\title{
Immune sensing of nucleic acids in inflammatory skin diseases
}

\author{
Olivier Demaria • Jeremy Di Domizio • Michel Gilliet
}

Received: 20 August 2014 / Accepted: 20 August 2014 / Published online: 16 September 2014

(C) Springer-Verlag Berlin Heidelberg 2014

\begin{abstract}
Endosomal and cytosolic nucleic acid receptors are important immune sensors required for the detection of infecting or replicating viruses. The intracellular location of these receptors allows viral recognition and, at the same time, avoids unnecessary immune activation to self-nucleic acids that are continuously released by dying host cells. Recent evidence, however, indicates that endogenous factors such as anti-microbial peptides have the ability to break this protective mechanism. Here, we discuss these factors and illustrate how they drive inflammatory responses by promoting immune recognition of self-nucleic acids in skin wounds and inflammatory skin diseases such as psoriasis and lupus.
\end{abstract}

\section{Introduction}

The skin is the largest organ of the human body. One of its main functions is the protection of internal organs from external insults. In addition to providing mechanical and chemical protection, the skin contains numerous resident immune cells that patrol the tissue under steady state conditions and recruit additional cells from the blood in the context of injury or infection. A first line of immune defense of the skin is provided by cells of the innate immune system. These innate immune cells express germ-line-encoded pathogen recognition receptors (PRRs) specialized in the detection of pathogens. Activation of PRRs triggers dendritic cell activation and maturation as well as cytokine production leading to inflammatory responses in the skin that clear infections and restore

This article is a contribution to the special issue on Mechanisms of Tissue Injury in Autoimmune Diseases - Guest Editor: Dan Eilat

O. Demaria $\cdot$ J. Di Domizio $\cdot$ M. Gilliet $(\bowtie)$

Department of Dermatology, Lausanne University Hospital CHUV, Lausanne, Switzerland

e-mail: michel.gilliet@chuv.ch homeostasis. PRRs recognize pathogen-associated molecular patterns (PAMPs) which are of lipid, protein, or nucleic acid origin. Whereas lipid and protein PAMPs are highly specific for pathogens, the discrimination of pathogen-derived from host-derived (self) nucleic acids is less clear. Indeed, there is increasing evidence that, under certain circumstances, nucleic acid receptors can be activated by host-derived self-DNA and self-RNA. Such recognition appears to be involved in skin inflammation in response to wounding but may also be central in the initiation and maintenance of chronic inflammatory skin diseases such as psoriasis and lupus.

\section{Nucleic acid receptors}

During evolution, the immune system has developed an efficient machinery to detect infections. Nucleic acid receptors that sense viral genomes (viral DNA and RNA, single- or double-stranded) represent a main component of this system. Nucleic acid receptors are commonly divided in two subgroups based on their endosomal or cytosolic distribution. In the endosomes, nucleic acid receptors include Toll-like receptor (TLR) 3, TLR7, TLR8, and TLR9. Viral nucleic acids can access endosomal compartments through scavenger receptor-mediated endocytosis and phagocytosis or Fc receptor-mediated uptake of the infecting viruses. In addition, viral nucleic acids can be re-directed from the cytosol to endosomes by the process of autophagy, a mechanism that allows TLR recognition of replicating viruses. In the cytosol, nucleic acid receptors include the retinoic acid-inducible gene I (RIGI)-like receptors, the cytosolic DNA sensors and receptors involved in inflammasome formation. Cytosolic receptors typically detect viral nucleic acids that accumulate in the cytoplasm during viral replication. 


\section{Endosomal Toll-like receptors}

The expression pattern of endosomal TLRs is different among immune cells allowing a specialization of each cell type at recognizing various endocytosed microbes. In humans, TLR7 and TLR9 expression is restricted to plasmacytoid dendritic cells (pDCs) and B cells, TLR8 expression is found in monocytes and conventional dendritic cells (cDCs), and TLR3 is expressed by cDCs [1].

\section{TLR9}

TLR9 senses unmethylated CpG-containing DNA motifs which are abundant in microbial DNA [2]. TLR9 activation leads to the formation of a multiprotein signaling complex composed of the adaptor molecule myeloid differentiation primary response protein 88 (MyD88) adaptor molecule. Activation of the MyD88 complex leads to the activation of $I \kappa B$ kinase (IKK) complex/nuclear factor- $\mathrm{KB}(\mathrm{NF}-\mathrm{kB})$ and mitogen-activated kinases (MAPKs)/activator protein 1 (AP1) pathways to induce proinflammatory responses. TLR9 triggering also induces the nuclear translocation of interferon regulatory factor 7 (IRF7) that leads to the production of type I interferons (IFNs). To note, human pDCs, the main type I IFN producers of the human body, harbor a unique and original spatiotemporal regulation of the MyD88-IRF7 pathway that allows retention of TLR9 and TLR7 ligands in endosomes and subsequent robust type I IFN induction [3] [4] (Fig. 1). Synthetic oligodeoxynucleotides (ODN) have been designed to specifically stimulate TLR9, including monomeric CpG ODN type B (CpG-B) and multimeric CpG ODN type A (CpG-A).

\section{TLR7 and TLR8}

TLR7 and TLR8 sense single-stranded RNA [5, 6]. Similarly to TLR9, they signal through MyD88 to activate the NF-KB and IRF7 pathways leading to the production of proinflammatory cytokines and type I IFN, respectively. Synthetic ligands for TLR7 and TLR8 have been designed, among which imiquimod is a selective agonist of TLR7.

\section{TLR3}

TLR3 recognizes viral double-stranded RNA [7], an intermediate product of viral replication. TLR3 signals through the TIR-domain-containing adaptor protein inducing IFN- $\beta$ (TRIF) that in turn activates the TANK-binding kinase 1 (TBK1)/interferon regulatory factor 3 (IRF3) pathway to produce type I IFN and also activates IKK complex/NF- $\mathrm{kB}$ to induce the production of proinflammatory cytokines [8]. Polyinosine-polycytidylic acid (poly(I:C)), a synthetic analog of double-stranded RNA (dsRNA), is used as a ligand for TLR3.

\section{Cytosolic DNA sensors}

Several cytosolic DNA sensors have been recently identified. These include IFI16, DAI, cyclic GMP-AMP synthase (cGAS), and RNA polymerase III, which all signal through stimulator of interferon gene (STING) and TBK1-IRF3. Others, such as Ku70, DHX9/DHX36, and AIM2, induce different signaling pathways. STING is a transmembrane protein of the endoplasmic reticulum, which upon activation triggers TBK1-dependent phosphorylation of IRF3 to initiate type I IFN production [9] [10] [11]. STING-deficient mice have been shown to present altered type I IFN production upon bacterial, viral, and mammalian DNA challenge [9]. In addition, STING can directly interact with bacterial cyclic dinucleotides c-di-AMP and c-di-GMP, suggesting that it can be considered a bona fide cytosolic DNA sensor [12].

\section{IFI16}

IFI16 belongs to the PYHIN protein family called HIN-200 in human and IFI200 in mice. IFI16 binds both ssDNA and double-stranded DNA (dsDNA) [13] and utilizes two distinct signaling pathways. On one hand, IFI16 can recruit ASC to form an inflammasome that triggers maturation and secretion of IL-1 $\beta$ and IL-18 [14]. On the other hand, IFI16 can promote STING recruitment and subsequent activation of the TBK1-IRF3 signaling pathway to produce type I IFNs [13].

\section{$D A I$}

The DNA-dependent activator of IFN-regulatory factors (DAI) recognizes cytosolic dsDNA and induces type I IFN in a TBK1-IRF3-dependent manner [15]. It also activates NF- $\mathrm{KB}$ via the receptor-interacting proteins 1 and 3 (RIP1, RIP3) [16]. Most probably, DAI transmits the signal through STING although this has not been formally demonstrated [18].

$c G A S$

Cyclic GMP-AMP synthase (cGAS) is a nucleotidyltransferase that forms an oligomeric complex with cytosolic DNA [17-19]. Upon dsDNA ligation, cGAS can generate the second messenger cGAMP from GTP and ATP [20], which physically interacts and activates STING [21-23], leading to type I IFN production via TBK1/IRF3 activation [24]. Cells deficient in cGAS do not produce type I IFN after infection by HIV, murine leukemia virus, or simian immunodeficiency virus [25]. In addition, cGAS-deficient mice are more susceptible to herpes simplex virus-1 (HSV-1) infection in comparison with WT mice [26]. 


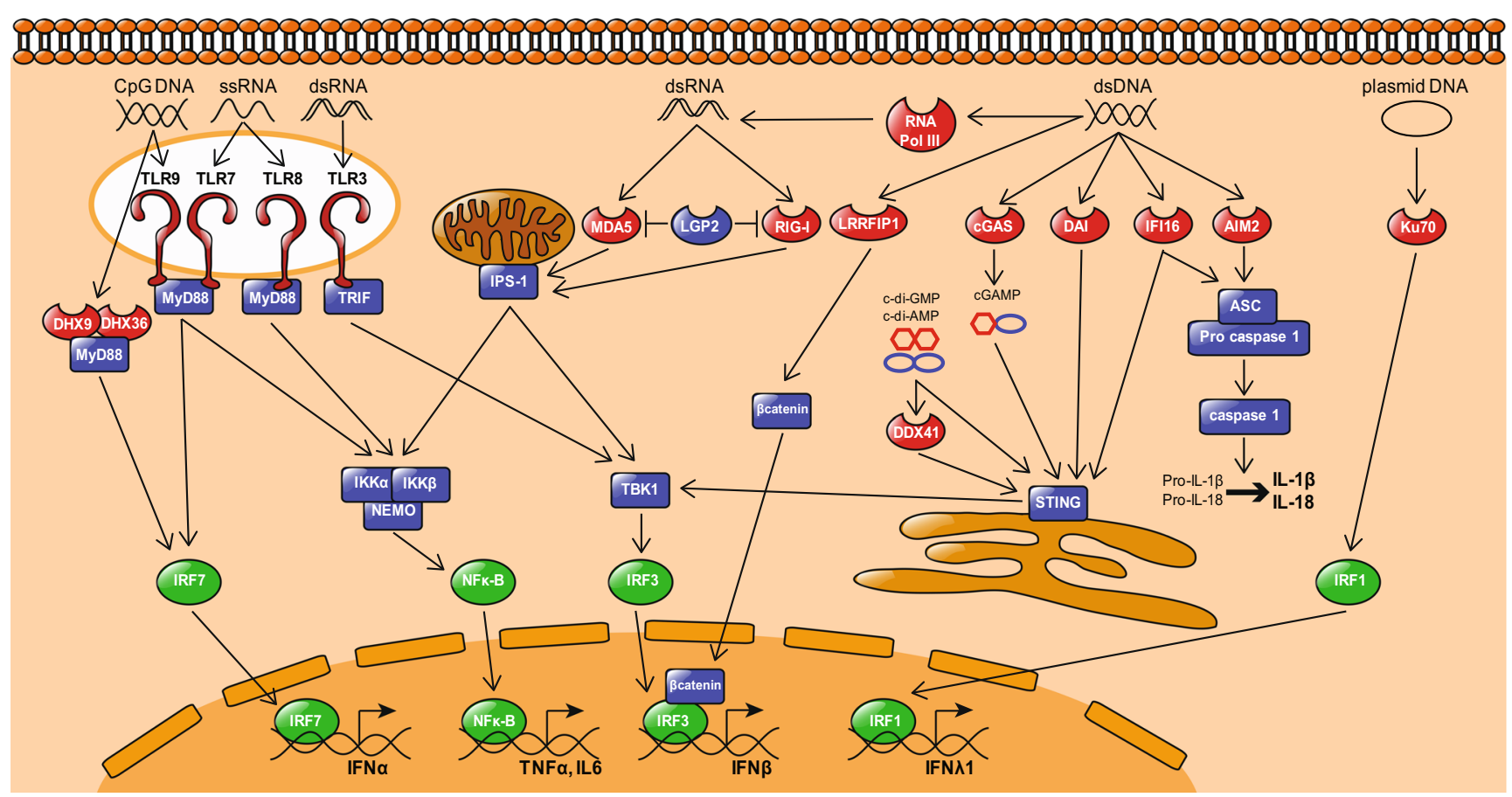

Fig. 1 Nucleic acids and their immune sensors. Multiple receptors allow the cell to sense the presence of microbial nucleic acids and to induce the production of proinflammatory cytokines. In endosomes, TLR9 senses the presence of CpG-containing DNA whereas TLR7 and TLR8 recognize ssRNA, leading to activation of the IKK complex via the MyD88 adaptor molecule and the subsequent activation of the NF- $\mathrm{kB}$ pathway, inducing the transcription of proinflammatory cytokines like TNF- $\alpha$ or IL6. TLR9 and TLR7 triggering also induces the translocation of IRF7 into the nucleus to initiate the expression of IFN- $\alpha$. The endosomal TLR3 recognizes dsRNA and uses the adaptor molecule TRIF to induce the TBK1-dependent activation of IRF3 and the subsequent transcription of the type I IFN- $\beta$. In addition to endosomal recognition, cytosolic receptors can sense the presence of nucleic acids in the cytoplasm. IFI16 and AIM2 sense dsDNA and recruit the adaptor protein ASC to form an inflammasome complex that activates the caspase 1 enzyme, leading to the processing and release of IL1 $\beta$ and IL18. Similarly to the dsDNA sensor DAI, IFI16 also activates the ER-associated adaptor molecule STING that induces the nuclear translocation of IRF3 via TBK1 and the transcription of IFN- $\beta$. LRRFIP1 potentiates IRF3 activity by activating $\beta$-catenin upon dsDNA sensing. Recently, dsDNA-activated cGAS was shown to catalyze cGAMP synthesis from GTP and ATP to induce STING-TBK1-IRF3-dependent IFN- $\beta$ expression. Other cyclic nucleotides (i.d. c-di-GMP and c-di-AMP) produced by bacteria were

DDX41 is another sensor that recognizes cyclic nucleotides. DDX41 contains a DEAD (Asp-Glu-Ala-ASP) box domain, which has been identified as a receptor for the bacterial second messengers cyclic-di-GMP or cyclic-di-AMP [27].

\section{Ku70}

$\mathrm{Ku} 70$ belongs to the heterodimeric $\mathrm{Ku}$ complex made of $\mathrm{Ku} 70$ and $\mathrm{Ku} 80 . \mathrm{Ku}$ complex is involved in nuclear processes such as in non-homologous end-joining pathway of DNA repair [28], V(D)J recombination, and telomerase maintenance but can also function as a receptor for DNA sensing by inducing described to activate the STING signaling pathway following recognition by the DDX41 helicase. The RNA polymerase III converts dsDNA into dsRNA to permit its recognition by MDA-5 and RIG-I. Both sensors signal through the mitochondria-associated IPS-1 that leads to the activation of the NF-KB and TBK1-IRF3 pathways and the expression of proinflammatory cytokines and IFN- $\beta$, respectively. The LGP2 RNA helicase is thought to compete with MDA-5 and RIG-I and inhibit their downstream signaling. Furthermore, new DNA helicases have been added to the cytosolic nucleic acids sensing machinery. Ku70 recognizes the presence of plasmid DNA and activates IRF1 to induce IFN $\lambda 1$ expression, and DHX9 and DHX36 are able to sense CpG DNA in the cytosol to induce the transcription of IFN- $\alpha$ via the MyD88-IRF7 pathway. TLR Toll-like receptor, $M y D 88$ myeloid differentiation primary response gene $88, I R F$ IFN-regulatory factor, TRIF TIR-domain-containing adaptor-inducing interferon- $\beta$, TBK1 TANK-binding kinase 1 , IFI1 6 IFN- $\gamma$-inducible protein 16, AIM2 absent in melanoma 2, DAI DNAdependent activator of IRF, LRRFIP1 leucine-rich repeat (in FLII) interacting protein $1, c G A S$ cyclic GMP-AMP synthase, $c G A M P$ cyclic guanosine monophosphate-adenosine monophosphate, $D D X$ DEAD-box protein, $D H X$ DEAH-box protein, $M D A-5$ melanoma differentiation-associated protein 5, RIG-I retinoic acid-inducible gene 1, LGP2 laboratory of genetics and physiology 2

the production of type III IFN via the activation and nuclear translocation of IRF1 and IRF7 [29].

\section{DHX9 and DHX36}

DHX9 and DHX36 are helicases containing a DExD/H domain-like RIG-I-like receptors (RLRs) that can sense CpG-containing DNA sequences in pDCs. DHX9 binds to $\mathrm{CpG}-\mathrm{B}$ and induces the production of proinflammatory cytokines through MyD88-dependent NF-kB activation. DHX36 recognizes $\mathrm{CpG}-\mathrm{A}$ and is associated with type I IFN production via MyD88-dependent nuclear translocation of IRF7 [30]. 
DHX9 has also been shown to participate in type I IFN production after dsRNA activation in myeloid DC in an IPS1-dependent manner [31].

\section{AIM2}

AIM2 preferentially binds dsDNA due to conserved motifs facilitating DNA binding [32] [33, 34]. Similarly to IFI16, after binding cytosolic dsDNA, AIM2 forms an inflammasome by recruiting $\mathrm{ASC}$ and caspase 1 to induce IL-1 $\beta$ and IL-18 maturation and secretion $[33,34]$.

\section{Cytosolic RNA sensors}

RIG-like receptors RLRs are cytosolic pattern recognition receptors broadly expressed by immune and non-immune cells and specialized in sensing RNA viruses. They belong to the $\mathrm{DExD} / \mathrm{H}$-box helicase superfamily comprising RIGI, melanoma differentiation-associated protein 5 (MDA5), and laboratory of genetics and physiology 2 (LGP2). RIG-I recognizes viral dsRNA [35] with a preference for the 5'-triphosphate part of the viral RNA [36]. In-vivo RIG-I is essential for the detection of several viruses such as Sendai virus and vesicular stomatitis virus. MDA-5 preferentially senses long dsRNA molecules [37] and is needed to detect picornavirus [38] and measles virus. However, RIG-I and MDA-5 may also allow detection of small host-derived self-RNA generated by the cytosolic ribonuclease RNAse L [39]. The exact contribution of LGP2 to cytosolic RNA sensing remains unclear, but crystal structure and NMR studies predict that LGP2 is likely to interact with a variety of RNA species [40-42]. Upon activation, RLRs signal through their caspase activation and recruitment domain (CARD) by binding to the IFN- $\beta$-promoter stimulator 1 (IPS1, aka MAVS) [8], an adaptor molecule that in turn activates IRFs and NF- $\mathrm{BB}$ transcription factors leading to the production of type I IFNs and proinflammatory cytokines. LGP2 lacks CARDs and thus is also considered as a negative regulator of RIGI and MDA-5.

\section{RNA polymerase III}

The DNA-dependent RNA polymerase III has been described as a B-form dsDNA sensor. It can transcribe AT-rich DNA sequence in a promoter-independent manner into 5'pppRNA that in turn can activate RIG-I-dependent pathway and induce type I IFN production [43, 44]. RNA polymerase III is required for IFN- $\beta$ induction by intracellular bacteria and DNA viruses such as Legionella pneumophila, adenovirus, HSV-1, or Epstein-Barr virus (EBV) [44].

\section{LRRFIP1}

Leucine-rich repeat flightless-interacting protein 1 (LRRFIP1) directly binds to exogenous dsRNA and B- or Z-form dsDNA to enhance IFN- $\beta$ gene expression. LRRFIP1 uses a unique signaling pathway since it binds $\beta$-catenin to promote its activation. Subsequently, $\beta$-catenin activates IRF3 that facilitates the recruitment of p300 acetyltransferase to the IFN- $\beta 1$ promoter. p300 acetyltransferase increases hyperacetylation of histones $\mathrm{H} 3$ and $\mathrm{H} 4$ and thus enhances IFN- $\beta$ expression [45]. LLRFIP1 has been shown to participate in the nucleic acid sensing of vesicular stomatitis virus and Listeria monocytogenes by macrophages [45].

\section{Discrimination between microbial and self-nucleic acids}

Despite the efficient recognition of microbial nucleic acids by intracellular nucleic acid receptors, self-nucleic acids (selfDNA and self-RNA) released into the extracellular environment by dying host cells typically remain immunologically inert. Three distinct mechanisms are in place to ensure responses to microbial nucleic acids and, at the same time, avoid unnecessary sensing of self-nucleic acids:

1. Chemical differences between pathogen and self-nucleic acids. TLR9 recognizes preferentially unmethylated CpG motifs, which are abundant in pathogen-derived DNA. However, it has become clear that self-DNA also contains a certain degree of unmethylated $\mathrm{CpG}$ islets that could activate TLR9. Furthermore, the phosphodiesteric backbone present in both pathogen-derived and host-derived self-DNA was shown to be a direct ligand for TLR9 [46]. RIG-I recognizes 5'-triphosphates present in viral RNA, which are usually masked or absent in self-RNA [36]. However, cytosolic DNA sensors recognize B-form DNA, which is common to both pathogen-derived and self-DNA independently of its sequence or methylation status. It becomes clear, therefore, that other mechanisms must be in place to avoid a continuous and unnecessary sensing of extracellular self-nucleic acids.

2. Rapid extracellular degradation of free nucleic acids by nucleases. Nucleic acids released into the extracellular environment by dying cells are rapidly eliminated via degradation by tissue nucleases. As a result, extracellular self-nucleic acids fail to gain access to immune cells that express nucleic acid sensors. Accordingly, deficiency of the DNA exonuclease Trex 1 allows internalization and cytosolic accumulation of DNA, leading to uncontrolled STING activation [47-49]. Furthermore, mice lacking DNAse II develop an inflammatory syndrome due to uncontrolled STING activation [50, 51]. 
3. Intracellular sequestration of nucleic acid receptors. The intracellular (endosomal or cytosolic) location of nucleic acid receptors allows sensing of microbial nucleic acids transported into intracellular compartments during the infectious process. By contrast, self-nucleic acids released in the extracellular environment by dying cells fail to spontaneously enter cells. An elegant study has demonstrated that enforced cell surface expression of TLR9 triggers uncontrolled sensing of self-nucleic acids with development of autoimmunity [52].

\section{Breaking innate tolerance to self-nucleic acids}

In the past few years, it has become clear that under certain circumstances, these protective mechanisms can be circumvented resulting in the conversion of otherwise nonstimulatory self-nucleic acids into potent activators of innate immunity. These circumstances are associated with the development of autoimmunity in psoriasis and lupus erythematosus and are linked to the expression of several endogenous factors including anti-microbial peptides, high-mobility group box 1 (HMGB1)/RAGE, autoantibodies, and amyloid precursors.

\section{Cationic anti-microbial peptides}

Cationic anti-microbial peptides (AMPs) belong to the first line of defense of the skin against bacterial, viral, and fungal invasion [53]. There are many different AMPs including the cathelicidins and the defensins, but they are all amphiphatic, containing a patch of positive charge clustered on one side of the molecule. This unique feature of AMPs confers them with antibiotic activity through binding of negatively charged phospholipids leading to the disruption of the microbial membrane. This feature also allows AMPs to form complexes with extracellular human nucleic acid fragments (DNA and RNA) and to form aggregated particles that are protected from extracellular degradation. In addition, exposed cationic residues in these particles allow the attachment of the nucleic acid complexes to heparin sulfate proteoglycans on the cell membrane leading to endocytosis of the complexes. Finally, the nucleic acid complexes gain access to endosomal compartments where they trigger activation of TLR7 and TLR9 in pDCs and TLR8 in myeloid dendritic cells (mDCs) [54, 55]. In addition, AMP-nucleic acid complexes can also be transported into the cytosol to activate STING/TBK1dependent cytosolic DNA sensors in monocytes [56]. Recent evidence suggests that the unique structural features of nucleic acid-AMP complexes permit interdigitation of endosomal TLRs, contributing to the strong efficiency of innate activation to self-nucleic acids.

\section{HMGB1 and RAGE}

HMGB1 is an important DNA binding protein that is present in the nucleus of cells where it regulates DNA accessibility to transcription factors [57]. HMGB1 is released into the extracellular environment during cell necrosis [58], where it may bind to free DNA through two DNA binding HMG box domains. HMGB1-DNA complexes were shown to induce type I IFN production by $\mathrm{pDCs}$ and to activate autoreactive $\mathrm{B}$ cells via TLR9 [59]. The receptor for advanced glycation end products (RAGE) is a receptor for HMGB1, although its role as a scavenger for DNA-HMGB1 complexes has not been demonstrated [60]. Interestingly, there is evidence that RAGE can directly bind nucleic acids, raising the possibility that it functions as a transporter itself [61].

\section{Amyloid fibrils}

Amyloid fibrils are stable insoluble aggregates of misfolded protein products with extensive $\beta$-sheet structures [62], involved in the pathogenesis of human diseases like Alzheimer's disease. Interestingly, these amyloid precursors are able to interact with nucleic acids [63] and to form complexes that protect DNA or RNA from degradation. Similarly to anti-microbial-nucleic acid complexes, complexes of amyloid precursors with DNA can be taken up by immune cells such as pDCs to trigger endosomal TLR9, leading to the production of type I IFN [64]. Although the link between the production of amyloid precursors and nucleic acidmediated innate immune responses needs to be fully elucidated, an interesting study has demonstrated that beta amyloid in Alzheimer's disease can trigger inflammasome activation [65], potentially via AIM2.

\section{Autoantibodies to DNA or RNA complexes}

In certain human autoimmune diseases, autoantibodies directed against nucleic acids and associated proteins like histones or ribonucleoproteins (RNPs) are being produced. These autoantibodies bind nucleic acids released by dying cells or released in the context of NETosis. On B cells, DNA or RNA containing immune complexes can bind to their specific BCR and be internalized to reach endosomal compartments where they can activate TLR7 or TLR9 signaling to induce autoreactive B cell proliferation [66, 67]. On antigenpresenting cells, DNA or RNA containing immune complexes can also bind to low affinity Fc $\gamma$ Rs that recognize the $\mathrm{Fc}$ fragment of antibodies and be engulfed within cells by endocytosis or phagocytosis to activate TLR7, TLR8, or TLR9. Such mechanisms have been demonstrated in $\mathrm{mDCs}$ [68] and pDCs [69-72]. In addition, the presence of AMPs such as LL37 in autoantibody complexes enhances immunogenicity of self-DNA [71, 72]. It is likely that aggregation and 
protection of self-nucleic acids induced by AMPs (described earlier) promote nucleic acid stability and cell internalization via the process of receptor-mediated endocytosis.

\section{Role of nucleic acid sensing in skin inflammation and autoimmunity}

\section{Skin wounds}

Following skin injury, an inflammatory response is mounted to prevent infection and to initiate the re-epithelialization process. Wounded human skin was found to contain large numbers of activated $\mathrm{pDCs}$, which specifically express TLR7 and TLR9 [1, 73-75]. A mouse model of skin injury indeed demonstrated that pDCs infiltrate injured skin and are activated to produce type I IFNs via TLR7 and TLR9. pDCderived type I IFNs were found to be crucial in driving the inflammatory response and the re-epithelialization process in wounded skin [73], although the link between type I IFNs and wound healing remains to be fully elucidated. Thus, sterile skin injury triggers TLR7 and TLR9 activation of pDCs, suggesting that this process is mediated via sensing of selfnucleic acids. But how are pDCs activated in the context of skin injury? One possibility is that AMPs produced by injured keratinocytes break innate tolerance to self-nucleic acids released into the wounds by dying cells. In accordance with this, AMPs are rapidly and transiently expressed in wounded skin, and their expression appears to parallel the IFN-expression by pDCs [73]. Another possibility is that neutrophils, which infiltrate injured skin along with pDCs, activate pDCs via the release of neutrophil extracellular traps (NETs) containing AMP-nucleic acid complexes (Fig. 2). This is in accordance with the finding that neutrophils in injured skin are activated to produce NETs and that NETs contain AMP-DNA complexes that activate pDCs.

\section{Psoriasis}

Psoriasis is a chronic inflammatory skin disease that affects 2$3 \%$ of the population worldwide and presents, in its most prevalent form, with scaly erythematous plaques. Psoriatic skin lesions are triggered by the aberrant activation of dermal dendritic cells that stimulate autoreactive Th17 cells. Activated Th17 cells migrate from the dermis into the epidermis and produce large amounts of Th17 cytokines, which induce keratinocyte activation with production of AMPs as well as the typical epidermal hyperproliferation that is characteristic of psoriatic plaques. Psoriatic lesions are also characterized by the presence of large numbers of activated pDCs [76]. In contrast to the transient infiltration and activation of pDCs in skin wounds, psoriatic pDCs were found to be chronically activated and to produce large amounts of type I IFNs that trigger unabated activation of dermal dendritic cells and autoreactive Th17 cells, leading to the development of the psoriatic plaque [76]. But what is the stimulus for pDC activation in psoriatic skin? Cationic AMPs such as LL37 and $\beta$ defensin, which are overexpressed in psoriatic skin, were found to constantly activate pDCs as well as conventional DCs by breaking innate tolerance to host nucleic acids. As described above, this process involves the complex formation between AMPs and free self-DNA and self-RNA, leading to activation of TLR7 and TLR9 in pDCs and TLR8 in conventional DCs [54, 55] (Fig. 2). So, whereas the transient AMP expression and $\mathrm{pDC}$ activation in skin wounds lead to an adequate inflammatory response that promotes reepithelialization of wounds, the constant AMP overexpression triggers sustained $\mathrm{pDC}$ activation and chronic inflammation that sustain keratinocyte hyperproliferation and development of the psoriatic plaque. But what are the mechanisms that stimulate chronic AMP overexpression in psoriatic skin? Th17 cytokines IL-17 and IL-22 are overexpressed in psoriatic skin due to an enhanced Th17 differentiation in psoriasis and can directly stimulate keratinocytes to produce AMPs [77, 78]. A genetic basis for the enhanced Th17 differentiation was revealed in the finding of IL-23 and IL-23R gene polymorphisms [79]. Furthermore, copy number polymorphisms in the $\beta$-defensin gene are directly associated with psoriasis, suggesting an additional link to AMP overexpression in psoriasis [80].

The role of endosomal TLRs in the triggering of psoriasis was further demonstrated by the observation that topical application of the TLR7 agonist imiquimod (Aldara $5 \%$ ) to the skin exacerbates human psoriasis [81] and the finding that repetitive application of Aldara to mouse skin induces a psoriasiform epidermal hyperproliferation [82, 83]. This process is driven by cDCs since depletion of CD11c-positive cells abrogates psoriasis development [83] and specific expression of MyD88 in dendritic cells of MyD88-deficient mice restores imiquimod-induced psoriasis [84]. Furthermore, specific depletion of ABIN1, a negative regulator of MyD88 and TLR signaling in the dendritic cell compartment, exacerbates psoriasis induced by imiquimod [85]. Similarly to the human disease, imiquimod model in mice shows strong involvement of the IL-23/IL-17 axis [84, 86]. Also, mice deficient in IL$17 \mathrm{a}, \mathrm{IL}-17 \mathrm{~F}, \mathrm{IL}-17 \mathrm{c}$, and IL-22 are somehow protected against imiquimod-induced psoriasis $[82,87,88]$, pointing out the role of these cytokines in this model. Whereas the source of Th17 cytokines in mice is gamma delta $\mathrm{T}$ cells $[83,86]$ and innate lymphoid cells [87], in humans, $\alpha \beta$-T lymphocytes seem to be the main cell type producing Th17 cytokines.

Lupus erythematosus

Lupus erythematosus (LE) is a complex autoimmune disease that primarily affects the skin but can become a multiorgan 


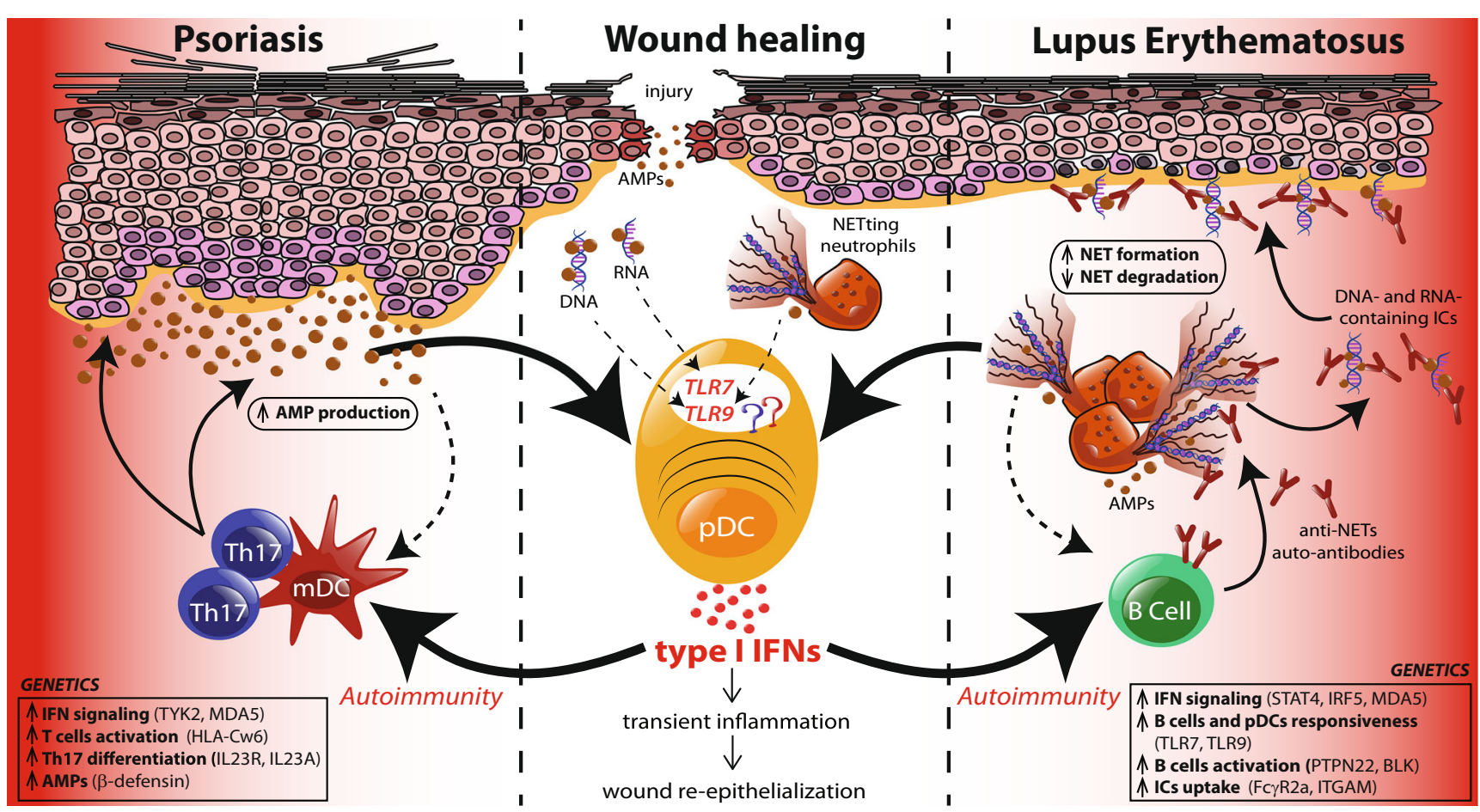

Fig. 2 TLR activation of pDCs in healthy and diseased skin. Upon skin injury, neutrophils first infiltrate the wound followed by pDCs. Antimicrobial peptides (AMPs) that are transiently produced by injured keratinocytes bind self-DNA and self-RNA released by dying cells and activate pDCs via TLR7 and TLR9. Neutrophil extracellular traps (NETs) extruded by neutrophils to prevent the spread of potential infection also trigger TLR9 in $\mathrm{pDCs}$. Activated $\mathrm{pDCs}$ produce type I IFNs that initiate a transient inflammation leading to the recruitment of additional immune cells that will participate in the re-epithelialization of the wound. Some genetic variations are associated with increased susceptibility to develop autoimmune diseases in response to environmental triggers. Psoriasis patients have a genetic predisposition that enhances the response to IFN signaling, reduces the threshold of $\mathrm{T}$ cell activation, and favors the differentiation of Th17 cells. Infiltrating Th17 cells produce cytokines, namely, IL-17 and IL-22, which increase the proliferation of

disease affecting the joints, heart, lungs, kidneys, and central nervous and hematologic systems [89]. Systemic LE is characterized by the presence of circulating immune complexes (ICs) formed by self-nucleic acids complexed with autoantibodies. These ICs are typically deposited in various tissues leading to detrimental inflammation that initiates and sustains autoimmunity. In the skin, ICs are deposited along the basal membrane of the epidermis and trigger a cytotoxic response leading to vacuolization of the basal keratinocyte layer. A key question in the pathogenesis of LE is the origin of ICs. In the skin, self-DNA may be released by dying cells during skin infection or ultraviolet light irradiation. LE patients were indeed found to have an intrinsic defect in clearing dying cells, leading to the accumulation of remnants containing self-nucleic acids [90]. Another source of self-DNA is neutrophil extracellular traps (NETs) released by neutrophils [71, 91]. NETs are filaments of nuclear DNA covered with granular proteins released by neutrophils during a cell death keratinocytes and their production of AMPs leading to enhanced stimulation of $\mathrm{pDCs}$ that sustain inflammation. Moreover, dermal DCs process AMPs as autoantigens to generate AMP-specific autoreactive T cells that perpetuate autoimmunity. Lupus patients have genetic variants that amplify IFN signaling and responsiveness of pDCs and B cells, leading to greater amounts of type I IFN that drive the generation of autoantibodies. Autoantibodies targeting NET-associated proteins and nucleic acids form immune complexes (ICs) that further deposit in the basal membrane of the dermo-epidermal junction of the skin and lead to keratinocyte necrosis and decreased epidermal thickness. Lupus patients also have a defect in the clearance of ICs which stimulates neutrophils to undergo NETosis. Increased NET formation and AMP production by neutrophils coupled to a defective NET degradation enhance $\mathrm{pDC}$ activation via TLR9 and favor the production of autoantibodies by B cells, sustaining the inflammatory response that participates in systemic autoimmunity

process called NETosis [92]. This process is dependent of the production of reactive oxygen species (ROS) and is due to the nuclear translocation of elastase, which decondenses nuclear DNA $[93,94]$. The expanding nuclear DNA breaks into the cytosol, mixes with the granular content of neutrophil, and is ultimately extruded as NETs into the extracellular space [94]. Interestingly, LE patients display increased numbers of NETs due to both enhanced formation [71, 72] as well as decreased nuclease-mediated degradation [95] (Fig. 2). These NETs were found to activate TLR9 in pDCs and trigger the production of type I IFNs [71].

Type I IFN production by pDCs is directly linked to disease exacerbation and maintenance of lupus. Indeed, a dominant type I IFN signature was identified in PBMC of SLE patients and was shown to correlate with disease activity and severity [96]. Whether activation of pDCs and their production of type I IFN can lead to the onset of the disease has been long debated. Recent work demonstrates that depletion of pDCs 
or treatment of mice with a bifunctional TLR7/TLR9 inhibitor prevents the development of skin lesions in lupus-prone mice [97]. Altogether, these data suggest that pDC activation seems to be an important step in the pathogenesis of lupus in these mice.

Several genetic studies that analyzed polymorphisms in TLR7, TLR8, and TLR9 genes in Caucasian and Asian populations revealed that some SNPs could be considered as SLE susceptibility factors $[98,99]$. It was shown that total PBMCs from SLE patients have higher expression of the TLR7 and TLR9 mRNAs compared to healthy donors that was positively correlated with IFN- $\alpha$ expression [100]. Increased expression of TLR7 in mice harboring duplication of the TLR7 gene is sufficient to drive the development of lupus, and deletion of this gene can improve the clinical disease [101]. By contrast, mouse models of deficiency for TLR9 and TLR8 demonstrated an exacerbation of the disease because of increased TLR7 response $[102,103]$. This was explained by a recent study showing that TLR8 controls TLR7 function on dendritic cells and TLR9 restrains TLR7 response on B cells [104]. Thus, alteration in the expression of TLR7, TLR8, or TLR9 can favor the loss of tolerance against self-nucleic acids. Importantly, injection of immunoregulatory sequences (IRSs) that inhibit both TLR7 and TLR9 signaling in lupus-prone mice can prevent the production of autoantibodies [105]. This dual inhibitor, called DV1179, is currently tested in a human clinical trial.

Autoreactive B cells produce antibodies targeting nucleic acids or associated proteins, including AMPs present in NETs that can be taken up by pDCs via Fc $\gamma$ RII leading to TLR7 and TLR9 activation [106, 107]. Anti-NETs antibodies such as anti-LL37 enhance NET formation by neutrophils through yet unclear mechanism [71]. This process provides an explanation for the sustained NETosis in SLE patients. Furthermore, antiNETs antibodies such as anti-DNA and anti-LL37 antibodies enhance IC uptake by pDCs, resulting in enhanced type I IFN production. Type I IFN production facilitates autoantibody production by autoreactive B cells that recognize ICs via TLR9 [67, 108]. Thus, autoantibodies targeting NETs enhance NETosis leading to the generation of autoreactive B cells, creating a self-sustaining loop that fuels the autoimmune response (Fig. 2).

Beyond TLRs, activation of other nucleic acid-sensing receptors may participate in the development of lupus. Strikingly, loss of STING function could inhibit self-DNA-induced inflammation in DNase II-deficient mice [51], suggesting that cytosolic DNA sensor pathways may be involved. Interestingly, an increased expression of HIN-200, the human gene encoding IFI16, was found in leukocytes of SLE patients and was correlated with anti-nuclear antibody titers [109]. Moreover, a strong sex bias was observed in the expression of IFI16 that correlates with a prevalence of women developing SLE [110]. However, the exact mechanism by which IFI16 is activated in LE is unknown. Furthermore, SLE PBMCs as well as lupus-prone mice have enhanced expression of DAI [111].

Genetic studies revealed that variants in MDA-5 are associated with autoantibody production in SLE patients [112] and increased type I IFNs [113]. Furthermore, a variant of IPS-1, an essential protein regulating MDA-5/RIG-I signaling, was associated with a subset of SLE patients [114]. Importantly, mutant mice harboring a constitutive activated MDA-5 spontaneously develop lupus-like autoimmune symptoms that can be ameliorated by intercrossing those mice with IFNARdeficient mice [115]. This supports the idea that cytosolic DNA and RNA recognition are also involved in lupus pathogenesis.

Furthermore, naïve mice injected with DNA-containing amyloids develop symptoms resembling lupus in a pDCdependent manner [64], indicating that amyloid precursors may also enhance self-DNA sensing in the course of lupus disease. Whether SLE patients have circulating amyloid precursors remains to be investigated. Intriguingly, neutrophil NETs appear to contain amyloidogenic proteins.

Other diseases with skin involvement related to cytosolic DNA sensors

Aicardi-Goutières syndrome (AGS) is a genetically determined inflammatory disease that develops in early childhood and manifests with inflammatory encephalopathy and SLElike skin lesions on the fingers, toes, and ears. Like SLE, AGS is associated with a strong type I IFN signature in PBMCs [116]. Mutations in nuclease genes including the exonuclease TREX1 (AGS1), the ribonucleases RNASEH2 (AGS2, AGS3, AGS4), and SAMHD1 (AGS5) have been identified $[117,118]$. These mutations lead to deficiency in nucleases and cellular accumulation of DNA or RNA leading to overactivation of cytosolic sensors and induction of type I IFN. As described earlier, mouse studies have shown that TREX1 deficiency drives STING activation and type I IFNinduced inflammation. Gain of function mutations in the MDA-5 gene has also been associated with the AGS, as well as other, yet undefined neurologic and immunological syndromes [119].

Recently, patients with mutations in the exon 5 of the TMEM173 gene, coding for the STING molecule, were identified. These patients presented cutaneous vasculitic lesions resembling AGS along with pulmonary inflammation. The syndrome was called STING-associated vasculopathy with onset in infancy (SAVI) [120]. STING is principally expressed by immune cells but is also widely expressed by fibroblast and endothelial cells. The mutation observed in SAVI leads to constitutive STING activation with spontaneous type I IFN production. As a major pathogenic event, STING activation of endothelial 
cells drives endothelial cell death and vascular damage through the excessive production of type I IFN [120].

\section{Conclusion and perspectives}

Nucleic acid receptors capable of detecting the presence of DNA and RNA in endosomes and cytosol have been recently identified. In addition to their role in anti-viral immunity, these receptors can, under certain circumstances, detect self-DNA and self-RNA released by dying host cells. Several endogenous factors enable immune recognition of self-nucleic acids. These factors include cationic AMPs, HMGB1 and its receptor RAGE, autoantibodies, and amyloid precursors. Dysregulated expression of these factors drives continuous sensing of self-nucleic acids and chronic nucleic acid receptor activation leading to autoimmunity and development of diseases like psoriasis and LE. Several key questions still remain to be answered: What controls the expression of these factors and why is there a dysregulated expression in psoriasis and LE? What is the role of structural cells of the skin in the recognition of self-nucleic acids? Psoriatic keratinocytes were found to express high levels of AIM2 and to produce high levels of IL$1 \beta$ in response to cytosolic DNA, raising the possibility for their contribution to the pathogenesis of psoriasis [121]. Furthermore, what is the factor that induces NETosis in both the injured healthy and diseased skin? As microbes can activate neutrophils to produce NETs, is there a role for the skin microbiome? Finally, is there a differential role of distinct AMPs or factors in targeting cell types or nucleic acid receptors? The answer to these questions will provide us with therapeutic targets to enhance wound healing or inhibit autoimmune inflammation of the skin.

\section{References}

1. Kadowaki N et al (2001) Subsets of human dendritic cell precursors express different toll-like receptors and respond to different microbial antigens. J Exp Med 194(6):863-869

2. Hemmi $\mathrm{H}$ et al (2000) A Toll-like receptor recognizes bacterial DNA. Nature 408(6813):740-745

3. Okuya K et al (2010) Spatiotemporal regulation of heat shock protein 90-chaperoned self-DNA and CpG-oligodeoxynucleotide for type I IFN induction via targeting to static early endosome. J Immunol 184(12):7092-7099

4. Guiducci $\mathrm{C}$ et al (2006) Properties regulating the nature of the plasmacytoid dendritic cell response to Toll-like receptor 9 activation. J Exp Med 203(8):1999-2008

5. Hemmi $\mathrm{H}$ et al (2002) Small anti-viral compounds activate immune cells via the TLR7 MyD88-dependent signaling pathway. Nat Immunol 3(2):196-200
6. Heil F et al (2004) Species-specific recognition of single-stranded RNA via toll-like receptor 7 and 8. Science 303(5663):1526-1529

7. Alexopoulou L et al (2001) Recognition of double-stranded RNA and activation of NF-kappaB by Toll-like receptor 3. Nature 413(6857):732-738

8. Kawai T, Akira S (2010) The role of pattern-recognition receptors in innate immunity: update on Toll-like receptors. Nat Immunol 11(5): 373-384

9. Ishikawa H, Ma Z, Barber GN (2009) STING regulates intracellular DNA-mediated, type I interferon-dependent innate immunity. Nature 461(7265):788-792

10. Zhong B et al (2008) The adaptor protein MITA links virus-sensing receptors to IRF3 transcription factor activation. Immunity 29(4): $538-550$

11. Tanaka Y, Chen ZJ, 214 (2012) STING specifies IRF3 phosphorylation by TBK1 in the cytosolic DNA signaling pathway. Sci Signal 5:ra20

12. Burdette DL et al (2011) STING is a direct innate immune sensor of cyclic di-GMP. Nature 478(7370):515-518

13. Unterholzner L et al (2010) IFI16 is an innate immune sensor for intracellular DNA. Nat Immunol 11(11):997-1004

14. Kerur N et al (2011) IFI16 acts as a nuclear pathogen sensor to induce the inflammasome in response to Kaposi Sarcomaassociated herpesvirus infection. Cell Host Microbe 9(5): 363-375

15. Takaoka A et al (2007) DAI (DLM-1/ZBP1) is a cytosolic DNA sensor and an activator of innate immune response. Nature 448(7152):501-505

16. Rebsamen $M$ et al (2009) DAI/ZBP1 recruits RIP1 and RIP3 through RIP homotypic interaction motifs to activate NF-kappaB. EMBO Rep 10(8):916-922

17. Zhang $X$ et al (2014) The cytosolic DNA sensor cGAS forms an oligomeric complex with DNA and undergoes switch-like conformational changes in the activation loop. Cell Rep 6(3):421-430

18. Li X et al (2013) Cyclic GMP-AMP synthase is activated by double-stranded DNA-induced oligomerization. Immunity 39(6): 1019-1031

19. Civril $F$ et al (2013) Structural mechanism of cytosolic DNA sensing by cGAS. Nature 498(7454):332-337

20. Gao $P$ et al $(2013)$ Cyclic $\left[\mathrm{G}\left(2^{\prime}, 5^{\prime}\right) \mathrm{pA}\left(3^{\prime}, 5^{\prime}\right) \mathrm{p}\right]$ is the metazoan second messenger produced by DNA-activated cyclic GMP-AMP synthase. Cell 153(5):1094-1107

21. Ablasser A et al (2013) cGAS produces a 2'-5'-linked cyclic dinucleotide second messenger that activates STING. Nature 498(7454):380-384

22. Zhang X et al (2013) Cyclic GMP-AMP containing mixed phosphodiester linkages is an endogenous high-affinity ligand for STING. Mol Cell 51(2):226-235

23. Diner EJ et al (2013) The innate immune DNA sensor cGAS produces a noncanonical cyclic dinucleotide that activates human STING. Cell Rep 3(5):1355-1361

24. Sun L et al (2013) Cyclic GMP-AMP synthase is a cytosolic DNA sensor that activates the type I interferon pathway. Science 339(6121):786-791

25. Gao D et al (2013) Cyclic GMP-AMP synthase is an innate immune sensor of HIV and other retroviruses. Science 341(6148):903-906

26. Li XD et al (2013) Pivotal roles of cGAS-cGAMP signaling in antiviral defense and immune adjuvant effects. Science 341(6152): 1390-1394

27. Parvatiyar K et al (2012) The helicase DDX41 recognizes the bacterial secondary messengers cyclic di-GMP and cyclic di-AMP to activate a type I interferon immune response. Nat Immunol 13(12):1155-1161

28. Walker JR, Corpina RA, Goldberg J (2001) Structure of the Ku heterodimer bound to DNA and its implications for double-strand break repair. Nature 412(6847):607-614 
29. Zhang X et al (2011) Cutting edge: Ku70 is a novel cytosolic DNA sensor that induces type III rather than type I IFN. J Immunol $186(8): 4541-4545$

30. Kim T et al (2010) Aspartate-glutamate-alanine-histidine box motif (DEAH)/RNA helicase A helicases sense microbial DNA in human plasmacytoid dendritic cells. Proc Natl Acad Sci U S A 107(34): 15181-15186

31. Zhang $Z$ et al (2011) DHX9 pairs with IPS-1 to sense doublestranded RNA in myeloid dendritic cells. J Immunol 187(9): $4501-4508$

32. Albrecht M, Choubey D, Lengauer T (2005) The HIN domain of IFI-200 proteins consists of two OB folds. Biochem Biophys Res Commun 327(3):679-687

33. Hornung $\mathrm{V}$ et al (2009) AIM2 recognizes cytosolic dsDNA and forms a caspase-1-activating inflammasome with ASC. Nature 458(7237):514-518

34. Burckstummer T et al (2009) An orthogonal proteomic-genomic screen identifies AIM2 as a cytoplasmic DNA sensor for the inflammasome. Nat Immunol 10(3):266-272

35. Yoneyama $\mathrm{M}$ et al (2004) The RNA helicase RIG-I has an essential function in double-stranded RNA-induced innate antiviral responses. Nat Immunol 5(7):730-737

36. Hornung $\mathrm{V}$ et al (2006) 5'-Triphosphate RNA is the ligand for RIGI. Science 314(5801):994-997

37. Kato $\mathrm{H}$ et al (2008) Length-dependent recognition of doublestranded ribonucleic acids by retinoic acid-inducible gene-I and melanoma differentiation-associated gene 5. J Exp Med 205(7): $1601-1610$

38. Gitlin L et al (2006) Essential role of mda-5 in type I IFN responses to polyriboinosinic:polyribocytidylic acid and encephalomyocarditis picornavirus. Proc Natl Acad Sci U S A 103(22):8459-8464

39. Malathi K et al (2007) Small self-RNA generated by RNase L amplifies antiviral innate immunity. Nature 448(7155):816-819

40. Murali A et al (2008) Structure and function of LGP2, a DEX(D/H) helicase that regulates the innate immunity response. J Biol Chem 283(23):15825-15833

41. Pippig DA et al (2009) The regulatory domain of the RIG-I family ATPase LGP2 senses double-stranded RNA. Nucleic Acids Res 37(6):2014-2025

42. Takahasi K et al (2009) Solution structures of cytosolic RNA sensor MDA5 and LGP2 C-terminal domains: identification of the RNA recognition loop in RIG-I-like receptors. J Biol Chem 284(26): $17465-17474$

43. Ablasser A et al (2009) RIG-I-dependent sensing of poly(dA:dT) through the induction of an RNA polymerase III-transcribed RNA intermediate. Nat Immunol 10(10):1065-1072

44. Chiu YH, Macmillan JB, Chen ZJ (2009) RNA polymerase III detects cytosolic DNA and induces type I interferons through the RIG-I pathway. Cell 138(3):576-591

45. Yang $P$ et al (2010) The cytosolic nucleic acid sensor LRRFIP1 mediates the production of type I interferon via a beta-catenindependent pathway. Nat Immunol 11(6):487-494

46. Haas $T$ et al (2008) The DNA sugar backbone $2^{\prime}$ deoxyribose determines toll-like receptor 9 activation. Immunity 28(3):315-323

47. Stetson DB et al (2008) Trex1 prevents cell-intrinsic initiation of autoimmunity. Cell 134(4):587-598

48. Yan $\mathrm{N}$ et al (2010) The cytosolic exonuclease TREX1 inhibits the innate immune response to human immunodeficiency virus type 1 . Nat Immunol 11(11):1005-1013

49. Morita $\mathrm{M}$ et al (2004) Gene-targeted mice lacking the Trex1 (DNase III) $3^{\prime}-5^{\prime}$ DNA exonuclease develop inflammatory myocarditis. Mol Cell Biol 24(15):6719-6727

50. Kawane $\mathrm{K}$ et al (2001) Requirement of DNase II for definitive erythropoiesis in the mouse fetal liver. Science 292(5521):15461549
51. Ahn J et al (2012) STING manifests self DNA-dependent inflammatory disease. Proc Natl Acad Sci U S A 109(47):19386-19391

52. Barton GM, Kagan JC, Medzhitov R (2006) Intracellular localization of Toll-like receptor 9 prevents recognition of self DNA but facilitates access to viral DNA. Nat Immunol 7(1):49-56

53. Zasloff M (2002) Antimicrobial peptides of multicellular organisms. Nature 415(6870):389-395

54. Ganguly D et al (2009) Self-RNA-antimicrobial peptide complexes activate human dendritic cells through TLR7 and TLR8. J Exp Med 206(9):1983-1994

55. Lande R et al (2007) Plasmacytoid dendritic cells sense self-DNA coupled with antimicrobial peptide. Nature 449(7162):564-569

56. Chamilos $\mathrm{G}$ et al (2012) Cytosolic sensing of extracellular selfDNA transported into monocytes by the antimicrobial peptide LL37. Blood 120(18):3699-3707

57. Stott $\mathrm{K}$ et al (2006) Structure of a complex of tandem HMG boxes and DNA. J Mol Biol 360(1):90-104

58. Scaffidi P, Misteli T, Bianchi ME (2002) Release of chromatin protein HMGB1 by necrotic cells triggers inflammation. Nature 418(6894):191-195

59. Tian $\mathrm{J}$ et al (2007) Toll-like receptor 9-dependent activation by DNA-containing immune complexes is mediated by HMGB1 and RAGE. Nat Immunol 8(5):487-496

60. Sirois CM et al (2013) RAGE is a nucleic acid receptor that promotes inflammatory responses to DNA. J Exp Med 210(11): 2447-2463

61. Sims GP et al (2010) HMGB1 and RAGE in inflammation and cancer. Annu Rev Immunol 28:367-388

62. Sideras K, Gertz MA (2009) Amyloidosis. Adv Clin Chem $47: 1-44$

63. Di Domizio J et al (2012) Binding with nucleic acids or glycosaminoglycans converts soluble protein oligomers to amyloid. J Biol Chem 287(1):736-747

64. Di Domizio J et al (2012) Nucleic acid-containing amyloid fibrils potently induce type I interferon and stimulate systemic autoimmunity. Proc Natl Acad Sci U S A 109(36):14550-14555

65. Halle A et al (2008) The NALP3 inflammasome is involved in the innate immune response to amyloid-beta. Nat Immunol 9(8):857-865

66. Leadbetter EA et al (2002) Chromatin-IgG complexes activate B cells by dual engagement of IgM and Toll-like receptors. Nature 416(6881):603-607

67. Lau CM et al (2005) RNA-associated autoantigens activate B cells by combined B cell antigen receptor/Toll-like receptor 7 engagement. J Exp Med 202(9):1171-1177

68. Boule MW et al (2004) Toll-like receptor 9-dependent and independent dendritic cell activation by chromatinimmunoglobulin G complexes. J Exp Med 199(12):1631-1640

69. Means TK et al (2005) Human lupus autoantibody-DNA complexes activate DCs through cooperation of CD32 and TLR9. J Clin Invest 115(2):407-417

70. Lovgren $\mathrm{T}$ et al (2006) Induction of interferon-alpha by immune complexes or liposomes containing systemic lupus erythematosus autoantigen- and Sjogren's syndrome autoantigen-associated RNA. Arthritis Rheum 54(6):1917-1927

71. Lande R et al (2011) Neutrophils activate plasmacytoid dendritic cells by releasing self-DNA-peptide complexes in systemic lupus erythematosus. Sci Transl Med 3(73):73ra19

72. Garcia-Romo GS et al (2011) Netting neutrophils are major inducers of type I IFN production in pediatric systemic lupus erythematosus. Sci Transl Med 3(73):73ra20

73. Gregorio J et al (2010) Plasmacytoid dendritic cells sense skin injury and promote wound healing through type I interferons. J Exp Med 207(13):2921-2930

74. Liu YJ (2005) IPC: professional type 1 interferon-producing cells and plasmacytoid dendritic cell precursors. Annu Rev Immunol 23: 275-306 
75. Jarrossay D et al (2001) Specialization and complementarity in microbial molecule recognition by human myeloid and plasmacytoid dendritic cells. Eur J Immunol 31(11):3388-3393

76. Nestle FO et al (2005) Plasmacytoid predendritic cells initiate psoriasis through interferon-alpha production. J Exp Med 202(1): 135-143

77. Zheng $Y$ et al (2007) Interleukin-22, a $\mathrm{T}(\mathrm{H}) 17$ cytokine, mediates IL-23-induced dermal inflammation and acanthosis. Nature 445(7128):648-651

78. Liang SC et al (2006) Interleukin (IL)-22 and IL-17 are coexpressed by Th17 cells and cooperatively enhance expression of antimicrobial peptides. J Exp Med 203(10):2271-2279

79. Capon $F$ et al (2007) Sequence variants in the genes for the interleukin-23 receptor (IL23R) and its ligand (IL12B) confer protection against psoriasis. Hum Genet 122(2):201-206

80. Stuart PE et al (2012) Association of beta-defensin copy number and psoriasis in three cohorts of European origin. J Investig Dermatol 132(10):2407-2413

81. Gilliet $\mathrm{M}$ et al (2004) Psoriasis triggered by toll-like receptor 7 agonist imiquimod in the presence of dermal plasmacytoid dendritic cell precursors. Arch Dermatol 140(12):1490-1495

82. van der Fits L et al (2009) Imiquimod-induced psoriasis-like skin inflammation in mice is mediated via the IL-23/IL-17 axis. J Immunol 182(9):5836-5845

83. Tortola L et al (2012) Psoriasiform dermatitis is driven by IL-36mediated DC-keratinocyte crosstalk. J Clin Invest 122(11):39653976

84. Wohn $\mathrm{C}$ et al (2013) Langerin(neg) conventional dendritic cells produce IL-23 to drive psoriatic plaque formation in mice. Proc Natl Acad Sci U S A 110(26):10723-10728

85. Callahan JA et al (2013) Cutting edge: ABIN-1 protects against psoriasis by restricting MyD88 signals in dendritic cells. J Immunol 191(2):535-539

86. Cai Y et al (2011) Pivotal role of dermal IL-17-producing gammadelta T cells in skin inflammation. Immunity 35(4):596-610

87. Pantelyushin $\mathrm{S}$ et al (2012) Ror $\gamma \mathrm{t}+$ innate lymphocytes and gammadelta $\mathrm{T}$ cells initiate psoriasiform plaque formation in mice. J Clin Invest 122(6):2252-2256

88. Van Belle AB et al (2012) IL-22 is required for imiquimod-induced psoriasiform skin inflammation in mice. J Immunol 188(1):462469

89. Croker JA, Kimberly RP (2005) SLE: challenges and candidates in human disease. Trends Immunol 26(11):580-586

90. Munoz LE et al (2010) The role of defective clearance of apoptotic cells in systemic autoimmunity. Nat Rev Rheumatol 6(5):280-289

91. Kaplan MJ (2011) Neutrophils in the pathogenesis and manifestations of SLE. Nat Rev Rheumatol 7(12):691-699

92. Brinkmann Vet al (2004) Neutrophil extracellular traps kill bacteria. Science 303(5663):1532-1535

93. Fuchs TA et al (2007) Novel cell death program leads to neutrophil extracellular traps. J Cell Biol 176(2):231-241

94. Papayannopoulos V et al (2010) Neutrophil elastase and myeloperoxidase regulate the formation of neutrophil extracellular traps. J Cell Biol 191(3):677-691

95. Hakkim A et al (2010) Impairment of neutrophil extracellular trap degradation is associated with lupus nephritis. Proc Natl Acad Sci U S A 107(21):9813-9818

96. Baechler EC et al (2003) Interferon-inducible gene expression signature in peripheral blood cells of patients with severe lupus. Proc Natl Acad Sci U S A 100(5):2610-2615

97. Guiducci $C$ et al (2010) Autoimmune skin inflammation is dependent on plasmacytoid dendritic cell activation by nucleic acids via TLR7 and TLR9. J Exp Med 207(13):2931-2942

98. Lee YH et al (2012) Associations between TLR polymorphisms and systemic lupus erythematosus: a systematic review and meta-analysis. Clin Exp Rheumatol 30(2):262-265
99. dos Santos BP et al (2012) TLR7/8/9 polymorphisms and their associations in systemic lupus erythematosus patients from southern Brazil. Lupus 21(3):302-309

100. Komatsuda A et al (2008) Up-regulated expression of Toll-like receptors mRNAs in peripheral blood mononuclear cells from patients with systemic lupus erythematosus. Clin Exp Immunol 152(3):482-487

101. Fairhurst AM et al (2008) Yaa autoimmune phenotypes are conferred by overexpression of TLR7. Eur J Immunol 38(7):1971-1978

102. Wu X, Peng SL (2006) Toll-like receptor 9 signaling protects against murine lupus. Arthritis Rheum 54(1):336-342

103. Demaria O et al (2010) TLR8 deficiency leads to autoimmunity in mice. J Clin Invest 120(10):3651-3662

104. Desnues B et al (2014) TLR8 on dendritic cells and TLR9 on B cells restrain TLR7-mediated spontaneous autoimmunity in C57BL/6 mice. Proc Natl Acad Sci U S A 111(4):1497-1502

105. Barrat FJ et al (2007) Treatment of lupus-prone mice with a dual inhibitor of TLR7 and TLR9 leads to reduction of autoantibody production and amelioration of disease symptoms. Eur J Immunol 37(12):3582-3586

106. Bave U et al (2003) Fc gamma RIIa is expressed on natural IFNalpha-producing cells (plasmacytoid dendritic cells) and is required for the IFN-alpha production induced by apoptotic cells combined with lupus IgG. J Immunol 171(6):3296-3302

107. Lovgren $\mathrm{T}$ et al (2004) Induction of interferon-alpha production in plasmacytoid dendritic cells by immune complexes containing nucleic acid released by necrotic or late apoptotic cells and lupus IgG. Arthritis Rheum 50(6):1861-1872

108. Viglianti GA et al (2003) Activation of autoreactive B cells by $\mathrm{CpG}$ dsDNA. Immunity 19(6):837-847

109. Kimkong I, Avihingsanon Y, Hirankarn N (2009) Expression profile of HIN200 in leukocytes and renal biopsy of SLE patients by realtime RT-PCR. Lupus 18(12):1066-1072

110. Choubey D et al (2011) Emerging roles for the interferon-inducible p200-family proteins in sex bias in systemic lupus erythematosus. J Interferon Cytokine Res 31(12):893-906

111. Zhang W et al (2013) DNA-dependent activator of interferonregulatory factors (DAI) promotes lupus nephritis by activating the calcium pathway. J Biol Chem 288(19):13534-13550

112. Molineros JE et al (2013) Admixture mapping in lupus identifies multiple functional variants within IFIH1 associated with apoptosis, inflammation, and autoantibody production. PLoS Genet 9(2):e1003222

113. Robinson T et al (2011) Autoimmune disease risk variant of IFIH1 is associated with increased sensitivity to IFN-alpha and serologic autoimmunity in lupus patients. J Immunol 187(3):1298-1303

114. Pothlichet $\mathrm{J}$ et al (2011) A loss-of-function variant of the antiviral molecule MAVS is associated with a subset of systemic lupus patients. EMBO Mol Med 3(3):142-152

115. Funabiki $M$ et al (2014) Autoimmune disorders associated with gain of function of the intracellular sensor MDA5. Immunity 40(2):199-212

116. Lebon $P$ et al (2002) Interferon and Aicardi-Goutieres syndrome. Eur J Paediatr Neurol 6(Suppl A):A47-A53, discussion A55-8, A77-86

117. Rice $G$ et al (2007) Heterozygous mutations in TREX1 cause familial chilblain lupus and dominant Aicardi-Goutieres syndrome. Am J Hum Genet 80(4):811-815

118. Rice GI et al (2009) Mutations involved in Aicardi-Goutieres syndrome implicate SAMHD1 as regulator of the innate immune response. Nat Genet 41(7):829-832

119. Rice GI et al (2014) Gain-of-function mutations in IFIH1 cause a spectrum of human disease phenotypes associated with upregulated type I interferon signaling. Nat Genet 46(5):503-509

120. Liu Y et al (2014) Activated STING in a vascular and pulmonary syndrome. N Engl J Med 371:507-518

121. Dombrowski Y, 82 et al (2011) Cytosolic DNA triggers inflammasome activation in keratinocytes in psoriatic lesions. Sci Transl Med 3:82ra38 